\title{
Comparative analysis of clinical and laboratory methods for diagnosing streptococcal sore throat
}

\author{
Ana Gabriela P. dos Santos, ${ }^{1}$ Eitan N. Berezin ${ }^{2}$
}

\begin{abstract}
Objectives: Diagnosis and correct treatment of group A streptococcal sore throat is important particularly to prevent non-suppurative sequelae. Clinical findings continue to be used to differentiate streptococcal infection from viral sore throat. The American Academy of Pediatrics recommends that streptococcal sore throat diagnosis should always be performed by microbiological identification methods. The aim of this study is to evaluate the accuracy of clinical diagnosis in comparison with culture and rapid test.

Methods: Children aged 2 to 13 years who had received a clinical diagnosis of sore throat and sought treatment at the pediatric emergency unit of São Paulo Santa Casa were evaluated and those with clinical signs or viral infection were excluded. Clinical findings were recorded and swabs were taken for group A Streptococcus cultures and a Streptococcus rapid test.

Results: The culture was positive in $96(24.4 \%)$ of the 376 children evaluated. The presence of petechiae, purulent exudate and painful tonsils were more likely to occur in children with positive streptococcus cultures, however they exhibited low diagnostic accuracy. The doctors' subjective evaluation failed to identify $21 \%$ of positive cases and antibiotics were prescribed in $47 \%$ of negative cases, compared with 3 and $6 \%$, respectively, for the rapid test.
\end{abstract}

Conclusions: A microbiologic method is necessary for the correct prescription of antibiotics in children with streptococcal sore throat.

J Pediatr (Rio J). 2005;81(1):23-8: Group A Streptococcus, tonsillitis, pharyngitis, Streptococcus pyogenes.

\section{Introduction}

Upper respiratory tract infections (URTI) are a significant cause of seeking medical services in the pediatric age group. One of the most common URTI types is sore throat caused by either viruses or bacteria. Viral causes are more common, with rhinovirus and adenovirus predominating, each being

1. M.Sc. Assistant physician, Department of Pediatrics, Santa Casa de Misericórdia de São Paulo, SP, Brazil.

2. Ph.D. Chief of the Service of Pediatric Infectious Diseases, Santa Casa de Misericórdia de São Paulo. Chief of the Pediatric Clinic, Hospital Sanatorinhos Itapevi. Professor, School of Medicine, Santa Casa de São Paulo, SP, Brazil.

Financial support: The main author received a scholarship from CAPES while pursuing her Master's degree.

Manuscript received Mar 22 2004, accepted for publication Oct 272004.

Suggested citation: dos Santos AG, Berezin EN. Comparative analysis of clinical and laboratory methods for diagnosing streptococcal sore throat. J Pediatr (Rio J). 2005;81:23-8. present in 6 to $20 \%$ of cases. ${ }^{1}$ Sore throats caused by Streptococcus pyogenes, more simply described as Group A streptococcus (GAS), merit highlighting particularly because they are the only type of sore throats for which antibiotic therapy is definitively indicated, with the objective of preventing non-suppurative sequelae (in particular rheumatic fever). ${ }^{2}$

While sore throats caused by viruses are more prevalent, the syndrome is one of the oldest and most widespread examples of how antibiotics are prescribed in an unsuitable manner. Wannamaker, was already warning against excessive antibiotic use for sore throats in $1972 .{ }^{3} \mathrm{~A}$ study performed on Rhode Island in 1973 showed that then, despite GAS being responsible for just $17 \%$ of sore throats, antibiotics were prescribed in $87 \%$ of cases. ${ }^{4}$ The consequences of improper antimicrobial usage can already be felt in the growth of bacterial resistance among other 
agents of community infections (such as Streptococcus pneumoniae and Haemophilus influenzae). In the case of sore throats, rational antibiotic use is dependent solely on a precise answer to the following question, "Is this sore throat streptococcal or not?"

The symptoms and/or signs that characterize sore throats are not sufficient for differential diagnosis between viral and bacterial causes. If exanthema scarlatina is presented in its classic form it is highly indicative of bacterial disease, with a high positive predictive value, but it is, in fact, very rare. ${ }^{2-9}$ A recent meta-analysis, including several different studies and a total of 5,453 patients, took each symptom in isolation and was unable to identify any symptom that could alone diagnose streptococcal sore throat. ${ }^{8}$

Even a combination of signs and symptoms was unable to differentiate with certainty between viral and bacterial sore throats. For this reason, a number of different authorities, including the Committee on Rheumatic Fever, Endocarditis, and Kawasaki Disease of the American Heart Association, the American Academy of Pediatrics and the Infectious Diseases Society of America recommend that diagnosis of streptococcal sore throat in patients suspected for clinical and epidemiological reasons should be confirmed by means of microbiological tests. $2,8,10,11$ A recent study performed by McIsaac et al., concluded that antibiotic treatment based on either a rapid test or oropharynx culture positive for GAS could reduce unnecessary antibiotic usage for sore throats. ${ }^{12}$

Our objective with the present study was to compare the accuracy of clinical and microbiological diagnostic methods for the identification of streptococcal sore throat.

\section{Methods}

A prospective, observational study undertaken at the Children's Emergency Service of the Santa Casa de Misericórdia in São Paulo between April 2000 and September 2001 and approved by the Ethics and Research Committee of that hospital on 15 March 2000.

Initially all children aged 2 to 13 years (uncompleted) complaining of URTI (defined as the presence of a painful throat and evidence of inflammation of throat or tonsils on physical examination) were enrolled. Children were then excluded from this group if they presented symptoms with onset more than 7 days previously or who presented signs of viral respiratory infection (defined as the presence of: rhinorrhea, coryza, conjunctivitis, coughing and/or sneezing).

Demographic and clinical data (presence or absence of: painful throat, dysphagia, fever, palpable glands, painful glands, hyperemia, edema, exudate from the palatine tonsils and exanthema scarlatina) were recorded by the on-call physician or second-year resident who treated the patient together with the subjective response to the following question: "Would you treat this patient with antibiotics or not?" Twin swab samples were taken by the researcher (previously trained fro the procedure). One of the swabs was used immediately for rapid testing using the commercially-available kit QuickVuePlus Strep A (Quidell Corporation, San Diego, CA). The remaining swab was sent to the laboratory at the Medical Sciences Faculty of the Santa Casa in São Paulo for culture in blood agar at $5 \%$, incubated for 24 hours at $35^{\circ} \mathrm{C}$ and in aerobic conditions for 48 hours. Streptococcus pyogenes was identified in positive cultures by means of testing for sensitivity to Bacitracina and PYR.

The accuracy of clinical diagnosis and of the rapid test was analyzed taking the result of the culture as the gold standard, in other words, determining which cases were truly positive and truly negative. Analyses were performed to define sensitivity and specificity, positive and negative predictive values and positive and negative likelihood ratios (LR), since, in the opinion of some authors, this tool establishes the magnitude of change in the probability of the disease, i.e. it indicates by how many times a test will increase the pre-test probability of an individual presenting the disease. ${ }^{13,14}$ Analyses of statistical significance were performed using the chi-square method with a cutoff of $p<0.001$

\section{Results}

Four hundred and twenty-one children were originally included in the study. Data were not complete in 23 cases (information was missing about clinical status or contained confused and/or illegible information) and the results of the culture were not recorded in 20 cases (material went astray or material arrived at the laboratory contaminated). Two children were excluded for not meeting inclusion criteria. The remaining 376 children made up the study sample.

The results of cultures were positive for GAS in 92 patients and negative in 284 patients.

With respect of distribution by sex, we found that 204 (54\%) patients were female and $172(46 \%)$ patients were male. There was no difference in positivity with respect of sex.

With respect of the distribution of positive cases by age, the greatest level of occurrence was between 4 and 8 years of age.

We compared the occurrence of each symptom for the positive culture group and the negative culture group. The incidence of symptoms for each group is illustrated in Table 1 . The symptoms petechiae, exudate and painful glands were more frequent among the subset of children with positive cultures, with statistical significance $(p<0.001)$. The frequency of palpable glands was greater among the negative group.

Exanthema scarlatina was described in just two children, which corresponds to $2.1 \%$ of the positive cases and $0.5 \%$ of the total number of cases. As this incidence was extremely low it could not be included in the analysis.

Sensitivity, specificity, predictive values and LR were calculated for symptoms whose frequency was greater among positive cases with a statistically significant difference. The calculations were performed in isolation for each symptom. The resultant values are shown in Table 2. 
Table 1 - Frequency of each symptom for the positive culture group and the negative culture group

\begin{tabular}{lcc}
\hline Symptom & $\begin{array}{c}\text { Frequency } \\
\text { for the positive } \\
\text { culture group }\end{array}$ & $\begin{array}{c}\text { Frequency } \\
\text { for the negative } \\
\text { culture group }\end{array}$ \\
\hline Hyperemia & $90 \%$ & $88 \%$ \\
Edema & $73 \%$ & $71 \%$ \\
Fever & $93 \%$ & $93 \%$ \\
Dysphagia & $27 \%$ & $23 \%$ \\
Petechiae* & $32 \%$ & $11 \%$ \\
Painful glands* & $36 \%$ & $15 \%$ \\
Exudate* & $50 \%$ & $36 \%$ \\
Palpable glands & $46 \%$ & $57 \%$
\end{tabular}

* Statistically significant difference. Chi-square test with Yates's correction resulted in $p$ value of $<0.001$.

Table 2 - Sensitivity, specificity, positive and negative predictive values, and LR+ and LR- of petechiae, exudate and painful glands were calculated for positive cases

\begin{tabular}{lccc}
\hline & Petechiae & Exudate & $\begin{array}{c}\text { Painful } \\
\text { glands }\end{array}$ \\
\hline Sensitivity (\%) & 32 & 50 & 36 \\
Specificity (\%) & 89 & 64 & 85 \\
Positive predictive value & 49 & 31 & 43 \\
Negative predictive value & 80 & 80 & 80 \\
Positive LR & 2.98 & 1.38 & 2.33 \\
Negative LR & 0.77 & 0.78 & 0.76 \\
\hline
\end{tabular}

$\mathrm{LR}=$ likelihood ratio.
Figure 1 demonstrates positive cases identified by the subjective criteria of the treating doctor at the emergency service, by the rapid test and by culture, distributed by patient age.

Figure 2 demonstrates those cases that were diagnosed by the doctor or by the rapid test as positive, but for which the GAS culture was negative (improper antibiotic usage) and those cases which were diagnosed as negative by the doctor or the rapid test, but as positive by culture (missed positives).

Table 3 shows the data analysis by means of sensitivity, specificity, predictive value and LR calculations for each method (physician and rapid test).

\section{Discussion}

In our research, studying children aged 2 to 12 years, presenting at the Children's Emergency Service with a history, complaint or physical examination compatible with infectious sore throat, we found a $24 \%$ level of positive GAS cultures. This figure allows us to state that our observed frequency corresponds with that found by a number of other authors, primarily when these studies had points in common with ours, i.e. the populations originated from densely populated urban areas and had sought treatment at a service located within a University Hospital. 15,16

Previous studies performed in our region have demonstrated practically the same incidence of positive GAS cultures. 17,18

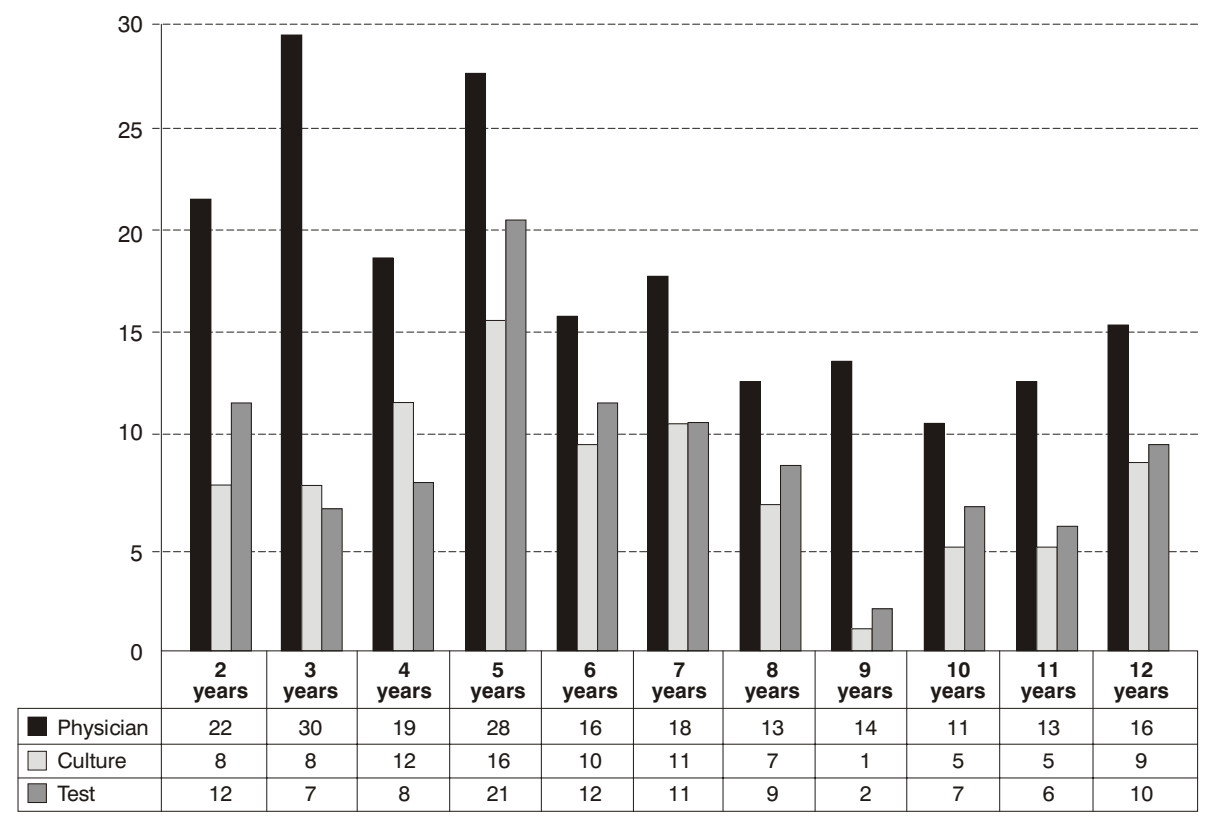

Figure 1 - Distribution of positive cases identified by the subjective criteria of the treating doctor at the emergency service, by the rapid test and by culture, distributed by patient age 


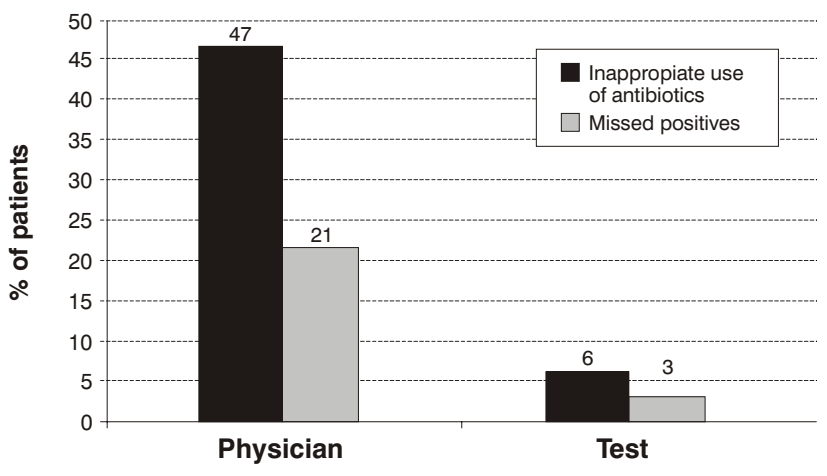

Figure 2 - Inappropiate use of antibiotics and missed positive cases by the doctor or the rapid test. The oropharynx culture determined the negative and positive cases of pharyngotonsillitis for group A Streptococcus

An analysis of the signs and symptoms that predominate with streptococcal sore throat showed statistically significant differences for: palatine petechiae, exudate and painful glands. Several studies have already attempted to establish a relation between the signs and symptoms of infectious sore throat and the presence of GAS. However, even for similar samples, the predominant signs and symptoms vary from study to study. Steinhoff et al. found statistically significant associations between positive cases and: exudate, fever above $38{ }^{\circ} \mathrm{C}$ and palpable glands. ${ }^{15} \mathrm{Nandi}$ et al., in India, found associations between enlarged tonsils, hyperemia and palpable glands. ${ }^{19}$

Cello et al. undertook a study in Brazil of a small sample of 51 children from 18 months to 13 years old and did not find any statistical significance for any symptom or sign, suggesting that clinical symptoms and data from physical examinations are insufficient for a precise diagnosis of bacterial sore throat and lead to errors in therapeutic conduct. ${ }^{20}$ Zuquim found statistical significance for odynophagia and exanthema scarlatina only. ${ }^{18}$

The difficulties involved in using clinical signs or symptoms in isolation to diagnose sore throats as streptococcal or not become even clearer when we analyze figures for sensitivity, specificity, positive and negative predictive values, LR positive and LR negative. Even with respect of symptoms that predominated among positive cases with statistical significance, the greatest positive predictive value is $50 \%$. The greatest LR value was an LR+ for the presence of petechiae of 2.98, which is considered capable of mildly altering the post-test probability and is therefore worthless for increasing diagnostic precision. None of the remaining LR values for the signs and symptoms in our study could be considered significant. A recently-performed meta-analysis found a that the highest LR value for any of the symptoms was 3.4 for exudate from the tonsils and concluded that, in isolation, no symptom was of use for diagnosing streptococcal sore throat. ${ }^{8}$

With respect of the subjective diagnoses performed by the physicians, it is possible to confirm observing Figure 1 that the accuracy of diagnosis of positive cases was low. At all ages the physicians identified many more positive cases than the rapid test or the culture. Analyzing Figure 2 it becomes obvious that diagnostic accuracy is also low for negative cases: $21 \%$ of the cases that were positive by culture were omitted in the diagnoses performed by the doctors.

The diagnostic accuracy of the subjective opinion of treating doctors has also been much studied. Breese \& Disney, in 1954 , found $69.6 \%$ accuracy for diagnoses made by doctors in a total of 11,999 patients. ${ }^{21}$ Attia et al. also assessed the subjective diagnostic capacity of doctors and concluded that $52 \%$ of patients without the disease would be treated and $28 \%$ of sick patients would not be offered treatment. ${ }^{9}$ In our study the physician would treat $47 \%$ of negative patients would fail to treat $21 \%$ of the positive patients, which values are very similar to those found by Attia et al. (Figure 2).

These analyses are reflected in the low sensitivity, specificity and positive and negative predictive values demonstrated in Table 3. The efficacy with which these criteria contribute to the exactitude of diagnosis, modifying the original expected probability of disease occurrence, is demonstrated by the LR values (Table 3 ). The LR represents the probability that a test is correct (positive or negative), or, in our case, of the method studied. An LR value has a

Table 3 - Sensitivity, specificity, positive and negative predictive values and posite and negative LR for the medical opinion (physician) and the rapid test, considering the culture result as the diagnostic definition

\begin{tabular}{lccccccc}
\hline & \multirow{2}{*}{ Sensitivity } & \multirow{2}{*}{ Specificity } & & \multicolumn{2}{c}{ Predictive value } & & \multicolumn{2}{c}{ LR } \\
& & & Positive & Negative & & Positive & Negative \\
\hline Physician & 79.3 & 53.2 & 35.43 & 88.8 & & 1.7 & 0.4 \\
Rapid test & 96.7 & 94.4 & 84.8 & 98.9 & & 17.2 & 0.03 \\
\hline
\end{tabular}

$\mathrm{LR}=$ likelihood ratio. 
strong modifying power when it is above 10 or below 0.1 , intermediate power when between 5 and 10 or 0.1 and 0.2 ; and weak between 2 and 5 or 0.2 a 0.5 . An LR value of 1.0 has no effect. In our study the analysis of diagnostic accuracy when the doctor identifies a case as positive or negative is, at best, weak. 13,14

With respect of the rapid test, values for sensitivity found by recent studies that analyzed tests with the same primary ingredient and from the same manufacturer that we used and with similar populations, are discretely inferior ( 91.2 to $95 \%) .22,23$

There are some possible explanations for the occurrence of false-positive test results: clinical differences between the study populations, the relation with low numbers of colonies on the cultures and the culturing method used as the gold standard.

With respect of the study populations it is possible that the fact that viral symptoms were considered as an exclusion criterion for the present study may have resulted in a reduced number of asymptomatic carriers in the sample. Asymptomatic carriers are generally not identified by rapid tests and perhaps this is why the observed sensitivity was higher.

The culturing was performed using the classic method. If one considers that we had 16 positive rapid tests with negative culture results the possibility, however remote, should be considered that, in fact, some of the cultures were "false-negatives". The possibility of "false-negative" cultures has primarily been demonstrated by comparing cultures grown using the classic method (ASB in aerobic incubation) with cultures grown on enriched media or incubated in other ways. ${ }^{24}$ The results of such studies are highly debatable.

In our country few studies have been undertaken with rapid tests. However, if the results found in our research were to be confirmed in similar populations, the use of this test in place of the classic oropharynx culture, with no requirement for confirmation of negatives could be ventured.

Nevertheless, if one takes the rapid test as the gold standard in our study and uses it to analyze the efficacy of the oropharynx cultures, a sensitivity of $84.7 \%$ is obtained together with a specificity of $98.9 \%$, a positive predictive value of $96.7 \%$, a negative predictive value of $94.3 \%$, an LR+ of 76 and an LR- of 0.15 . The LR+ and the LR- of such an analysis would be classed as very strong in terms of the power to modify pre-test probability, reasserting the efficacy of microbiological methods for the identification of streptococcal sore throat.

The high incidence of infection by GAS in Brazil, with the resulting presence of serious non-suppurative sequelae, allied to the constant threat of the appearance of multiresistant strains of bacteria responsible for community infections leads us to conclude that the appropriate management of these infections and prevention of sequelae involves correct treatment of a clinically suspected infection diagnosed in the laboratory.

The results returned lead us to suggest that the 1994 guidelines of the American Academy of Pediatrics, recommending the use of microbiological methods, preferably oropharynx cultures, be followed. These guidelines were reinforced in 1998 with the publication of criteria for the rational use of antibiotics. ${ }^{25}$

\section{Acknowledgements}

We are grateful to all the treating doctors and residents at the Santa Casa de Misericórdia Pediatrics Department in São Paulo who were involved in data collection.

Thanks also to the microbiology laboratory at the School of Medicine of Santa Casa de Misericórdia de São Paulo where the analysis of oropharynx cultures was carried out.

\section{References}

1. Middleton DB. Pharyngitis. Prim Car. 1996;23:719-39.

2. Bisno AL, Gerber MA, Gwaltney JM, Kaplan EL, Schwartz RH. Infectious Diseases Society of America. Practice guidelines for the diagnosis and management of group A streptococcal pharyngitis. Infectious Diseases Society of America. Clin Infect Dis. 2002;35:113-25.

3. Wannamaker LW. Perplexity and precision in the diagnosis of streptococcal pharyngitis. Am J Dis Child. 1972;124:352-8.

4. Holmberg SD, Faich GA. Streptococcal pharyngitis and acute rheumatic fever in Rhode Island. JAMA. 1983; 250:2307-12.

5. Breese BB, Disney FA, Talpey WB, Green JL. Beta-hemolytic streptococcal infection: the clinical and epidemiologic importance of the number of organisms found in cultures. Am J Dis Child. 1970;119:18-26.

6. Centor RM, Meier FA, Dalton HP. Throat cultures and rapid tests for diagnosis of group A Streptococcal pharyngitis. Ann Inter Med. 1986;105:892-9.

7. Kaplan EL. Clinical Guidelines for group A streptococcal throat infections. Lancet. 1997;350:899-900.

8. Ebell MH. Making decisions at the point of care: sore throat. Fam Pract Manag. 2003;10(8):68-9.

9. Attia MW, Zadutis T, Eppe S, Klein J. Performance of a predictive model for group A Beta-hemolytic Estreptococos pharyngitis in children. Acad Emerg Med. 1999;69:8-13.

10. Dajani A, Taubert K, Ferrieri P. Committee on Rheumatic Fever, Endocarditis, and Kawasaki Disease of the American Heart Association. Treatment of acute streptococcal pharyngitis and prevention of rheumatic fever: a statement for health professionals. Pediatrics. 1995;96:758-64.

11. American Academy of Pediatrics. Group A Streptococcal Infections. In: Pickering LK, ed. Red Book: 2003 Report of the Committee on Infections diseases. 26th ed. Elk Grove Village (IL): American Academy of Pediatrics; 2003. p. 573-84.

12. McIsaac WJ, Kellner JD, Aufricht $P$, Vanjaka A, Low DE. Empirical validation of guidelines for the management of pharyngitis in children and adults. JAMA. 2004;291:1587-95.

13. Jaeschke R, Guyatt GH, Sackett DL. How to use an article about a diagnostic test: $B$. What are the results and will they help me in caring for my patients? JAMA. 1994;271:703-7.

14. Hayden SR, Brown MD. Likelihood ratio: a powerful tool for incorporating the results of a diagnostic test into clinical decisionmaking. Ann Emerg Med. 1999;33:575-80.

15. Steinhoff MC, Abd El Khalek MK, Khallaf N, Hamza HS, El Ayadi $A$, Orabi $A$, et al. Effectiveness of clinical guidelines for the presumptive treatment of streptococcal pharyngitis in Egyptian children. Lancet. 1997;350:918-21.

16. Edmond KM, Grimwood K, Carlin JB, Chondros JB, Hogg GC, Barnett $P$. Streptococcal pharyngitis in a pediatric emergency department. Med J Austr. 1996;165:420-3.

17. Berezin EM, Massarato LC, Gazzeta RS, Designe R, Raphaelian T, Mimica IM, et al. Faringotonsilites estreptocócicas: diagnóstico clínico e laboratorial. Rev Paul Pediatr. 1996;14:177-9.

18. Zuquim SL. Diagnóstico clínico e laboratorial das faringotonsilites estreptocócicas na infância [dissertação]. São Paulo (SP): Faculdade de Ciências Médicas da Santa Casa de São Paulo; 1997. 
19. Nandi S, Kumar R, Ray P, Vohra H, Ganguly N.K. Clinical score card for diagnosis of group A streptococcal sore throat. Indian J Pediatr. 2002;69:471-5.

20. Cello AC, Macatti EF, Oliveira GD, Neves GR, Martins J, Siqueira LF, et al. Avaliação de amigdalite estreptocócica em crianças. Revista de Ciências Médicas. 1999;8:11-4.

21. Breese BB, Disney FA. The accuracy of diagnosis of beta streptococcal infections on clinical grounds. J Pediatr. 1954;44:670-3.

22. Spadetto CC, Camara SM, Ingles MJA, Escuriet JM, Barcelo IC, Sanchez FR. Rational use of antibiotics in pediatrics: impact of a rapid test for detection of beta-haemolytic group A streptococci in acute pharyngotonsillitis. An Esp Pediatr. 2000;52:212-9.

23. QUIDEL Corporation [homepage on the Internet]. San Diego: c2003 Quidel Corporation [cited 2005 Jan 17] Procedure manual QuickVue+StrepA test for use by health care professionals; [about 54 screens] Available from: http://www.quidel.com/ libraries/pkginserts/RD/QuickVuePlusStrepA.pdf.
24. Schroedes S, Procop GW. False positive strep A antigen test. Pediatr Infect Dis J. 2000;19:1114-5.

25. Schwartz B, Marcy SM, Philips WR, Gerber MA, Dowell SF. Pharyngitis: principle of judicious use of antimicrobial agents. Pediatrics. 1998;101:171-4.

Corresponding author:

Ana Gabriela Pires dos Santos

Av. Washington Luis, 1527/13, Bloco B

CEP 04662-002 - São Paulo, SP

Brazil

E-mail: gabipds@ig.com.br 